\title{
Introduction to Social Media: Culture, Identity, and Inclusion Minitrack
}

\author{
Shahper Vodanovich \\ Auckland University of \\ Technology, New Zealand \\ svodanov@aut.ac.nz
}

\author{
Nanette S. Levinson \\ American University \\ nlevins@american.edu
}

\author{
Amanda Menking \\ University of Washington, \\ Seattle amenking@uw.edu
}

It is fitting that the $50^{\text {th }}$ anniversary meeting of HICSS marks the initiation of this new minitrack related to social media. The focus on the intersections of digital and social media with culture, identity and, indeed, inclusion begins to fill an increasingly significant gap in our research, teaching, and practice. Digital and social media are now ubiquitous, and there has been much recent research related to social media and systems sciences. Yet oftentimes this work does not explore relations with culture, identity, or inclusion.

The knowledge niche of this minitrack as it connects to culture(s), identity (ies), and inclusion represents a range of research methods and conceptual frameworks. The papers presented at HICSS 50 serves as indicators of the richness of emerging research in this arena and, in some cases, build on research traditions dating back to the seventies (see, for example, the work of Hall (1976) or Hofstede (1980) on culture and cross-cultural communication, or Rogers (1962) on the diffusion of innovations including work that links identity to diffusion). Papers like "Detecting Offensive Statements towards Foreigners in Social Media" and "Social Media Usage and Cultural Dimensions: an Empirical Investigation" adeptly apply existing understandings of culture and the "Other" to new forms of media and engagement.

Looking ahead, technology-facilitated social media present opportunities for new frontiers of research both as they interact with cultures, identities, and diversity or inclusion, and also as the technology type changes over time-just as we are seeing a change from more traditional computer-mediated social media to mobile media and beyond. What shapes will the interactions take in the future, and what are the implications of such trajectories for research and practice? The paper "Using the Control Balance Theory to Explain Social Media Deviance" suggests ways to develop SM networks that are safe, supportive, responsible and constructive", while the paper "Gender (In)Consistent
Communication via Social Media and Hireability: An Exploratory Study" suggests the implications of using Social Media for hiring processes.

Systems sciences cover a range of contexts and settings including public, private, and not or profit sectors. In recent years, as documented in HICCS papers, there has been a tremendous growth in partnerships and alliances and in cross-national collaborations. How do we study social media in collaborative (and also competitive) contexts (including crowd-sourcing contexts), always cognizant of interactions with cultures and identities? For example, a paper like "Modifying Existing Aggression and Verbal Violence Scales for Measuring Harassment in Individual Tweets" presents negative results, challenging our assumptions about how "easy" it may be leverage existing measures to understand phenomenon specific to certain kinds of sociotechnical systems.

Additionally, we need to remember the culture kaleidoscope (e.g., the myriad levels of culture from small group and occupational, to organizational, interorganizational and national) as well as the intersections of culture and identity. Similar to the culture kaleidoscope there are also multiple and complex identities including cultural identities, age and generational identities, racial identities, and gender identities. Most importantly the intersections among social media and the culture and identity kaleidoscopes presage tremendous research opportunities as we examine social media usages (and concomitant outcomes) in myriad contexts. The paper "Mapping Articles on China in Wikipedia: An Inter-Language Semantic Network Analysis" is one such example that looks at how culture of different language groups influences the process of crowdsourcing knowledge on online collaboration platforms. Taken together this research sets the scene for understanding better how social media can foster or prevent diversity and inclusion in an increasingly local and global world. 